\title{
Estadística de egresos hospitalarios de la Secretaría de Salud, 2000
}

$\mathrm{P}$ ara el año 2000 la estadística de egresos hospitalarios de la Secretaría de Salud (SSA) ha tenido algunos cambios importantes, entre los que destacan el incremento en la cobertura de egresos, la incorporación del resto de los hospitales del Distrito Federal (con excepción de los Institutos Nacionales de Salud) al Sistema Automatizado de Egresos Hospitalarios (SAEH) y la substitución del catálogo de procedimientos quirúrgicos, utilizado hasta 1999, por el de Procedimientos en Medicina, el cual constituye un volumen adicional de la Modificación Clínica de la CIE-9. Este último es un listado más actualizado, de procedimientos quirúrgicos, diagnósticos y terapéuticos no quirúrgicos. La Clasificación Estadística Internacional de Enfermedades y Problemas Relacionados con la Salud, Décima Revisión (CIE10) se utiliza para la codificación de las enfermedades y otros motivos de atención, en tanto que la de procedimientos de la CIE-9 MC se utiliza para integrar la estadística de cirugías y actividades de diagnóstico y tratamiento médico.

Ambas clasificaciones permiten no sólo conocer de qué se atienden y fallecen los pacientes sino, además, qué tratamientos y métodos diagnósticos fueron aplicados durante el episodio atendido. La información generada es un auxiliar importante para analizar los motivos de demanda, los resultados de la atención, los costos, etcétera, y tiene implicaciones importantes para la planeación y, en general, para la gestión hospitalaria.

En esta ocasión, la Dirección General de Información y Evaluación del Desempeño de la SSA presenta un resumen de la morbilidad, mortalidad y procedimientos quirúrgicos registrados en las unidades hospitalarias de la institución, que proceden de dos fuentes principales: El SAEH y los sistemas de los Institutos Nacionales de Salud; el primero se aplica en $98 \%$ de los hospitales y capta $94 \%$ de los egresos, en tanto que a los segundos les corresponde el porcentaje restante.

La estadística de morbilidad y mortalidad abarca la totalidad de las instituciones, en tanto que la de procedimientos quirúrgicos sólo comprende a los hospitales que utilizan el SAEH, debido a que aún no se incorporan las cirugías de los Institutos Nacionales de Salud.

El cuadro I actualiza la cobertura de unidades, egresos y defunciones obtenidas a través de estos sistemas desde 1994 hasta el año 2000. Conviene destacar que algunos cambios bruscos en las cifras obedecen a varios aspectos:

Con el SAEH, hasta 1995 la estadística de egresos se obtuvo mediante muestreo con representatividad nacional, aunque prácticamente se captaba la totalidad de las defunciones, y es a partir de 1996, cuando se capta la totalidad de egresos y además se amplía paulatinamente la cobertura de hospitales.

Por otra parte, la información del Hospital General de México se incorporó a esta estadística en 1998.

En los cuadros II a V se muestra la distribución de egresos y defunciones por entidad federativa, según grupos de edad y principales servicios de atención. Los cuadros VI y VII muestran el orden y peso relativo de las principales causas de atención y de defunción hospitalaria, es decir, la afección principal y la causa básica. En ambos cuadros las causas ocupan más de $80 \%$ de los casos atendidos, por lo que brindan un excelente resumen de la demanda y la mortalidad.

Una información más detallada sobre algunas causas seleccionadas por su importancia epidemiológica según edad y sexo, se encuentra en los

* Sección preparada por la Dirección General de Información y Evaluación del Desempeño de la Secretaría de Salud, México. 
cuadros VIII a XI, en los cuales además se incluye una columna para el promedio de estancia por enfermedad.

Finalmente, los cuadros XII a XIV contienen información sobre los procedimientos quirúrgicos practicados en ese año, estadística relativamente nueva y de gran importancia porque también traduce costos. En esta ocasión se presenta agrupada en grandes capítulos por edad y sexo, destinando un cuadro final para mostrar los 20 principales procedimientos quirúrgicos que comprenden $80 \%$ de los casos.
Esta información combinada con la afección principal, la presencia de co-morbilidad y complicaciones es utilizada para la gerencia hospitalaria con el apoyo de tecnología como la de los Grupos de Diagnóstico Relacionados "GRDs", que ha sido recientemente incorporada por una institución de nuestro país.

Como en años anteriores, la SSA publica también el Anuario Estadístico respectivo, en donde el lector podrá consultar información más detallada por entidad y hospital, así como el Boletín de Información Es- tadística del Sistema Nacional de Salud, en donde se encuentra la información para las instituciones de todo el país. De igual modo, debe destacarse que la información depende mucho de la calidad y cuidado que los médicos tengan para registrar las enfermedades, traumatismos, otros motivos de atención y procedimientos practicados, así como del cuidado de los codificadores, los cuales con el desarrollo y uso de esta estadística han mejorado su confiabilidad.

Cuadro I

Egresos y defunciones en hospitales de LA SSA. México, 1994-2000

\begin{tabular}{|c|c|c|c|c|c|c|c|c|c|c|c|c|c|c|}
\hline \multirow{2}{*}{$\begin{array}{l}\text { Concepto } \\
\text { Unidades Informando } \\
\text { Total }\end{array}$} & \multicolumn{2}{|c|}{$\begin{array}{c}1994 \\
229\end{array}$} & \multicolumn{2}{|l|}{$\begin{array}{c}1995 \\
242\end{array}$} & \multicolumn{2}{|c|}{$\begin{array}{c}1996 \\
274\end{array}$} & \multicolumn{2}{|c|}{$\begin{array}{c}1997 \\
291 \\
\end{array}$} & \multicolumn{2}{|c|}{$\begin{array}{c}1998 \\
383 \\
\end{array}$} & \multicolumn{2}{|c|}{$\begin{array}{c}1999 \\
467\end{array}$} & \multicolumn{2}{|c|}{$\begin{array}{c}2000 \\
468\end{array}$} \\
\hline & Núm. & $\%$ & Núm. & $\%$ & Núm. & $\%$ & Núm. & $\%$ & Núm. & $\%$ & Núm. & $\%$ & Núm. & $\%$ \\
\hline esos & 954295 & 100.0 & 1048703 & 100.0 & 1017674 & 100.0 & 1131609 & 100.0 & 1270305 & 100.0 & 1372161 & 100.0 & 1469332 & 100.0 \\
\hline Hombres & 250239 & 26.2 & 284691 & 27.1 & 266739 & 26.2 & 307998 & 27.2 & 346311 & 27.3 & 377851 & 27.5 & 395431 & 26.9 \\
\hline Mujeres & 704056 & 73.8 & 760515 & 72.5 & 747747 & 73.5 & 812646 & 71.8 & 918868 & 72.3 & 994197 & 72.5 & 1073811 & 73.1 \\
\hline No especificado & 0 & 0.0 & 3497 & 0.3 & 3188 & 0.3 & 10965 & 1.0 & 5126 & 0.4 & 113 & 0.0 & 90 & 0.0 \\
\hline funciones & 27702 & 100.0 & 31378 & 100.0 & 26152 & 100.0 & 30956 & 100.0 & 35839 & 100.0 & 35891 & 100.0 & 36531 & 100.0 \\
\hline Hombres & 15627 & 56.4 & 17687 & 56.4 & 14372 & 55.0 & 16738 & 54.1 & 19889 & 55.5 & 19986 & 55.7 & 20203 & 55.3 \\
\hline Mujeres & 11987 & 43.3 & 13601 & 43.3 & 11655 & 44.6 & 13730 & 44.4 & 15894 & 44.3 & 15880 & 44.2 & 16317 & 44.7 \\
\hline No espec & 88 & 0.3 & 90 & 0.3 & 125 & 0.5 & 488 & 1.6 & 56 & 0.2 & 25 & 0.1 & 11 & 0.0 \\
\hline
\end{tabular}

SAEH*

$\begin{array}{llllllllllllllll}\text { Egresos } & 815313 & 100.0 & 891603 & 100.0 & 873498 & 100.0 & 977409 & 100.0 & 1106658 & 100.0 & 1192461 & 100.0 & 1375939 & 100.0\end{array}$

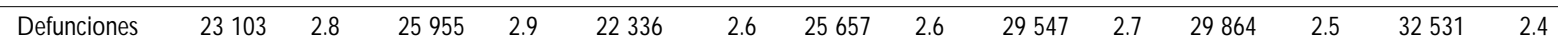

IN S. y HGM ${ }^{\ddagger}$

\begin{tabular}{|c|c|c|c|c|c|c|c|c|c|c|c|c|c|c|}
\hline Egresos & 34924 & 100.0 & 54502 & 100.0 & 53060 & 100.0 & 54964 & 100.0 & 87773 & 100.0 & 92641 & 100.0 & 93393 & 100.0 \\
\hline & 942 & 5.6 & 2072 & 3.8 & 1837 & 3.5 & 1966 & 3.6 & 3622 & 4.1 & 4019 & 4.3 & 4000 & \\
\hline
\end{tabular}

$S S A D D F^{\S}$

\begin{tabular}{lrrrrrrrrrrrrr} 
Egresos & 104058 & 100.0 & 102598 & 100.0 & 91116 & 100.0 & 99236 & 100.0 & 75874 & 100.0 & 87059 & 100.0 & $\S$ \\
\hline Defunciones & 2657 & 2.6 & 3351 & 3.3 & 1979 & 2.2 & 3333 & 3.4 & 2670 & 3.5 & 2008 & 2.3 & $\S$
\end{tabular}

Fuentes:

* Hospitales que usan el Sistema Automatizado de Egresos Hospitalarios en el país (SAEH)

₹ Institutos N acionales de Salud y a partir de 1998 se incluye al Hospital General de México

$\S$ Hospitales de Secretaría de Salud del DF (antiguamente del DDF). Para el 2000 se incorporan al sistema SAEH 
Cuadro II

Egresos Hospitalarios por edad según entidad federativa de atención. México, 2000

\begin{tabular}{|c|c|c|c|c|c|c|c|c|}
\hline \multirow[b]{2}{*}{ Entidad Federativa } & \multirow[b]{2}{*}{ Total } & \multicolumn{6}{|c|}{ Grupos de edad } & \multirow[b]{2}{*}{ NE } \\
\hline & & -1 & 1 a 4 & 5 a 14 & 15 a 44 & 45 a 64 & $65 y+$ & \\
\hline Estados Unidos Mexicanos & 1469332 & 127416 & 61063 & 88995 & 950519 & 141444 & 99676 & 219 \\
\hline Aguascalientes & 26924 & 2274 & 1194 & 1775 & 17121 & 2410 & 2150 & 0 \\
\hline Baja California & 27238 & 2488 & 659 & 1104 & 19390 & 2142 & 1431 & 24 \\
\hline Baja California Sur & 10485 & 814 & 355 & 485 & 7039 & 1008 & 784 & 0 \\
\hline Campeche & 17768 & 1678 & 722 & 1038 & 11623 & 1638 & 1067 & 2 \\
\hline Coahuila & 25252 & 1724 & 1367 & 1588 & 17070 & 2053 & 1450 & 0 \\
\hline Colima & 13288 & 1050 & 461 & 705 & 8680 & 1199 & 1188 & 5 \\
\hline Chiapas & 48996 & 4369 & 1663 & 2886 & 33372 & 4186 & 2520 & 0 \\
\hline Chihuahua & 16501 & 1294 & 830 & 1269 & 9139 & 2087 & 1882 & 0 \\
\hline Distrito Federal & 245773 & 27515 & 14455 & 20704 & 137028 & 28702 & 17187 & 182 \\
\hline Durango & 26480 & 2614 & 1054 & 1804 & 16102 & 2619 & 2287 & 0 \\
\hline Guanajuato & 67110 & 5193 & 2513 & 3399 & 48088 & 4509 & 3408 & 0 \\
\hline Guerrero & 45387 & 3840 & 2109 & 2968 & 29158 & 4055 & 3257 & 0 \\
\hline Hidalgo & 30117 & 2157 & 782 & 1412 & 20696 & 2960 & 2110 & 0 \\
\hline Jalisco & 74006 & 4085 & 2254 & 3806 & 52057 & 6281 & 5523 & 0 \\
\hline México & 126844 & 11201 & 3760 & 4883 & 89541 & 10584 & 6875 & 0 \\
\hline Michoacán & 58058 & 4456 & 2030 & 4032 & 37147 & 5809 & 4584 & 0 \\
\hline Morelos & 26211 & 2465 & 1187 & 1558 & 16766 & 2337 & 1897 & 1 \\
\hline $\bar{N}$ ayarit & 19542 & 1246 & 1087 & 1555 & 11840 & 1980 & 1834 & 0 \\
\hline Nuevo León & 37286 & 4913 & 1625 & 1544 & 24123 & 2820 & 2261 & 0 \\
\hline 0 axaca & 36071 & 3204 & 1459 & 2203 & 22953 & 3706 & 2546 & 0 \\
\hline Puebla & 41126 & 3082 & 1371 & 1893 & 28520 & 3607 & 2653 & 0 \\
\hline Q uerétaro & 25784 & 3117 & 866 & 1276 & 17424 & 1801 & 1300 & 0 \\
\hline Q uintana Roo & 14338 & 1326 & 466 & 715 & 10240 & 1036 & 555 & 0 \\
\hline San Luis Potosí & 24813 & 2674 & 902 & 1349 & 16340 & 1968 & 1580 & 0 \\
\hline Sinaloa & 43916 & 2527 & 1672 & 2623 & 27966 & 4885 & 4243 & 0 \\
\hline Sonora & 57442 & 4224 & 2644 & 3046 & 36920 & 7041 & 3567 & 0 \\
\hline Tabasco & 58699 & 4193 & 2600 & 4211 & 38699 & 5798 & 3193 & 5 \\
\hline Tamaulipas & 62599 & 5571 & 3173 & 3533 & 38809 & 6521 & 4992 & 0 \\
\hline Tlaxcala & 23627 & 2309 & 831 & 1152 & 16296 & 1797 & 1242 & 0 \\
\hline Veracruz & 95375 & 6362 & 3508 & 5936 & 62438 & 10145 & 6986 & 0 \\
\hline Yucatán & 20633 & 2080 & 703 & 1224 & 13681 & 1847 & 1098 & 0 \\
\hline Zacatecas & 21643 & 1371 & 761 & 1319 & 14253 & 1913 & 2026 & 0 \\
\hline
\end{tabular}

NE: no especificado

Fuente: Sistema Automatizado de Egresos Hospitalarios en el país (SAEH) y Sistemas de información de los Institutos N acionales de Salud y del Hospital General de México 


\section{Cuadro III}

\section{Egresos Hospitalarios por servicio según entidad federativa de atención. México, 2000}

\begin{tabular}{|c|c|c|c|c|c|c|}
\hline $\begin{array}{l}\text { Entidad Federativa } \\
\text { Unidad de Ocurrencia }\end{array}$ & Total & Medicina interna & Cirugía & Gineco-0 bstetricia & Pediatría & Otro \\
\hline Estados Unidos Mexicanos & 1469332 & 168677 & 215689 & 702170 & 213671 & 169125 \\
\hline Aguascalientes & 26924 & 3819 & 3359 & 13103 & 4278 & 2365 \\
\hline Baja California & 27238 & 3079 & 4006 & 15935 & 3714 & 504 \\
\hline Baja C alifornia Sur & 10485 & 1452 & 1912 & 5397 & 1144 & 580 \\
\hline Campeche & 17768 & 2200 & 2546 & 8977 & 2766 & 1279 \\
\hline Coahuila & 25252 & 3288 & 3862 & 14102 & 3301 & 699 \\
\hline Colima & 13288 & 1798 & 2328 & 7088 & 1866 & 208 \\
\hline Chiapas & 48996 & 6373 & 8639 & 26099 & 7726 & 159 \\
\hline Chihuahua & 16501 & 3519 & 4620 & 5271 & 2394 & 697 \\
\hline Distrito Federal & 245773 & 10172 & 24338 & 67843 & 34057 & 109363 \\
\hline Durango & 26480 & 3455 & 4677 & 11918 & 4976 & 1454 \\
\hline Guanajuato & 67110 & 6830 & 9012 & 40602 & 9727 & 939 \\
\hline Guerrero & 45387 & 4912 & 8439 & 23284 & 7978 & 774 \\
\hline Hidalgo & 30117 & 4085 & 3920 & 15955 & 3367 & 2790 \\
\hline Jalisco & 74006 & 8079 & 14798 & 41696 & 5866 & 3567 \\
\hline México & 126844 & 15813 & 17057 & 69332 & 17373 & 7269 \\
\hline Michoacán & 58058 & 6113 & 8244 & 28920 & 9984 & 4797 \\
\hline Morelos & 26211 & 2892 & 3686 & 13379 & 4402 & 1852 \\
\hline $\bar{N}$ ayarit & 19542 & 3602 & 2725 & 8970 & 3013 & 1232 \\
\hline Nuevo León & 37286 & 4162 & 3249 & 20380 & 7566 & 1929 \\
\hline 0 axaca & 36071 & 4065 & 6699 & 18008 & 6683 & 616 \\
\hline Puebla & 41126 & 3960 & 7075 & 22581 & 5362 & 2148 \\
\hline Q uerétaro & 25784 & 2203 & 3266 & 14804 & 4653 & 858 \\
\hline Q uintana Roo & 14338 & 1469 & 1857 & 8327 & 2009 & 676 \\
\hline San Luis Potosí & 24813 & 2206 & 2967 & 14235 & 4518 & 887 \\
\hline Sinaloa & 43916 & 7137 & 8815 & 20781 & 6766 & 417 \\
\hline Sonora & 57442 & 14159 & 8527 & 24124 & 4724 & 5908 \\
\hline Tabasco & 58699 & 8457 & 9538 & 27688 & 9354 & 3662 \\
\hline Tamaulipas & 62599 & 9629 & 9784 & 30024 & 10817 & 2345 \\
\hline Tlaxcala & 23627 & 2379 & 3093 & 13858 & 3209 & 1088 \\
\hline Veracruz & 95375 & 12399 & 16659 & 47407 & 14174 & 4736 \\
\hline Yucatán & 20633 & 2108 & 2835 & 10557 & 3784 & 1349 \\
\hline Zacatecas & 21643 & 2863 & 3157 & 11525 & 2120 & 1978 \\
\hline
\end{tabular}

En otro servicio se incluyen los egresos de los Institutos $\mathrm{N}$ acionales de Salud y Hospital General de México

Fuente: Sistema Automatizado de Egresos Hospitalarios en el país (SAEH) y Sistemas de información de los Institutos N acionales de Salud y del Hospital General de México 


\section{Cuadro IV}

Defunciones Hospitalarias por grupos de edad según entidad federativa. México, 2000

\begin{tabular}{|c|c|c|c|c|c|c|c|c|}
\hline \multirow{2}{*}{$\begin{array}{l}\text { Entidad Federativa } \\
\text { Unidad de Ocurrencia }\end{array}$} & \multirow[b]{2}{*}{ Total } & \multicolumn{6}{|c|}{ Grupos de edad } & \multirow[b]{2}{*}{$\mathrm{NE}$} \\
\hline & & -1 & 1 a 4 & 5 a 14 & 15 a 44 & 45 a 64 & $65 y+$ & \\
\hline Estados Unidos Mexicanos & 36531 & 9856 & 836 & 729 & 7048 & 7691 & 10350 & 21 \\
\hline Aguascalientes & 586 & 140 & 12 & 20 & 105 & 97 & 212 & 0 \\
\hline Baja California & 1065 & 270 & 12 & 13 & 272 & 231 & 262 & 5 \\
\hline Baja California Sur & 248 & 49 & 4 & 3 & 46 & 53 & 93 & 0 \\
\hline Campeche & 395 & 117 & 3 & 10 & 79 & 77 & 107 & 2 \\
\hline Coahuila & 385 & 82 & 5 & 2 & 56 & 75 & 165 & 0 \\
\hline Colima & 318 & 47 & 2 & 3 & 55 & 66 & 142 & 3 \\
\hline Chiapas & 1446 & 577 & 34 & 50 & 291 & 280 & 214 & 0 \\
\hline Chihuahua & 613 & 118 & 14 & 7 & 110 & 130 & 234 & 0 \\
\hline Distrito Federal & 7253 & 1325 & 238 & 212 & 1631 & 1882 & 1957 & 8 \\
\hline Durango & 650 & 141 & 10 & 13 & 94 & 131 & 261 & 0 \\
\hline Guanajuato & 1280 & 426 & 27 & 14 & 232 & 240 & 341 & 0 \\
\hline Guerrero & 1247 & 419 & 49 & 30 & 241 & 214 & 294 & 0 \\
\hline Hidalgo & 807 & 195 & 6 & 7 & 184 & 185 & 230 & 0 \\
\hline Jalisco & 944 & 143 & 9 & 9 & 141 & 179 & 463 & 0 \\
\hline México & 3362 & 925 & 40 & 27 & 665 & 783 & 922 & 0 \\
\hline Michoacán & 1466 & 477 & 29 & 36 & 260 & 263 & 401 & 0 \\
\hline Morelos & 799 & 214 & 25 & 12 & 125 & 177 & 245 & 1 \\
\hline $\mathrm{N}$ ayarit & 455 & 142 & 19 & 15 & 53 & 70 & 156 & 0 \\
\hline Nuevo León & 631 & 144 & 8 & 5 & 86 & 132 & 256 & 0 \\
\hline 0 axaca & 759 & 306 & 15 & 13 & 149 & 128 & 148 & 0 \\
\hline Puebla & 995 & 440 & 35 & 13 & 144 & 151 & 212 & 0 \\
\hline Q uerétaro & 730 & 228 & 22 & 14 & 161 & 139 & 166 & 0 \\
\hline Q uintana Roo & 296 & 129 & 7 & 3 & 57 & 40 & 60 & 0 \\
\hline San Luis Potosí & 445 & 163 & 15 & 10 & 51 & 77 & 129 & 0 \\
\hline Sinaloa & 1084 & 282 & 12 & 8 & 178 & 219 & 385 & 0 \\
\hline Sonora & 1032 & 251 & 18 & 12 & 158 & 224 & 369 & 0 \\
\hline Tabasco & 1455 & 564 & 51 & 52 & 323 & 234 & 229 & 2 \\
\hline Tamaulipas & 1610 & 362 & 32 & 24 & 272 & 354 & 566 & 0 \\
\hline Tlaxcala & 458 & 201 & 7 & 4 & 60 & 66 & 120 & 0 \\
\hline Veracruz & 2874 & 775 & 61 & 78 & 613 & 606 & 741 & 0 \\
\hline Yucatán & 481 & 122 & 4 & 4 & 97 & 110 & 144 & 0 \\
\hline Zacatecas & 362 & 82 & 11 & 6 & 59 & 78 & 126 & 0 \\
\hline
\end{tabular}

NE: no especificado

Fuente: Sistema Automatizado de Egresos Hospitalarios en el país (SAEH) y Sistemas de información de los Institutos N acionales de Salud y del Hospital General de México 


\title{
Cuadro V
}

\section{Defunciones hospitalarias por servicio según entidad federativa de atención. México, 2000}

Entidad Federativa

Unidad de Ocurrencia

\begin{abstract}
Total
\end{abstract}
Medicina interna

17346

36531

Cirugía

Estados Unidos Mexicanos

586

A guascalientes

Baja California Sur

Campeche

Coahuila

Colima

Chiapas

Chihuahua

Distrito Federal

Durango

Guanajuato

Guerrero

Hidalgo

Jalisco

México

Michoacán

Morelos

$\mathrm{N}$ ayarit

Nuevo León

0 axaca

Puebla

Q uerétaro

Q uintana Roo

San Luis Potosí

Sinaloa

Sonora

Tabasco

Tamaulipas

Tlaxcala

Veracruz

Yucatán

Zacatecas

En otro servicio se incluyen los egresos de los Institutos N acionales de Salud y Hospital General de México

Fuente: Sistema Automatizado de Egresos Hospitalarios en el país (SAEH) y Sistemas de información de los Institutos N acionales de Salud y del Hospital General de México 


\section{Principales causas de Morbilidad Hospitalaria, SSA. México, 2000}

Núm. de orden
Causa
Clave CIE-10

A $00-Y 98$

Egresos

$\%$

Total

Parto único espontáneo

080

0 10-075 081-092 096-087

C ausas obstétricas directas (excepto aborto)

Traumatismos envenenamientos y algunas otras consecuencias

de causas externas

- Fracturas

$4 \quad$ Aborto

$5 \quad$ Ciertas afecciones originadas en el periodo perinatal

- Dificultad respiratoria del recién nacido y otros trastornos respiratorios

originados en el periodo perinata

- Crecimiento fetal lento, desnutrición fetal y trastornos relacionados

con la gestación corta y el bajo peso al nacer

Tumores malignos

- Tumor maligno del cuello del útero

- Leucemias

- Tumor maligno de la mama

Influenza y N eumonía

Hernia de la cavidad abdominal

Colelitiasis y colecistitis

Enfermedades del apéndice

Diabetes mellitus

Enfermedades infecciosas intestinales

Enfermedades del corazón

Malformaciones congénitas, deformidades y anomalías cromosómicas

Insuficiencia renal

Infecciones respiratorias agudas

Atención para la anticoncepción

Leiomioma uterino

Enfermedades del hígado

Bronquitis crónica y la no especificada, enfisema y asma

Síntomas, signos y hallazgos anormales clínicos y de laboratorio no clasificados

en otra parte

R00-R99

16642

1.1

Las demás causas

273504

18.6

Clave CIE-10: códigos de la Clasificación Estadística Internacional de Enfermedades y Problemas Relacionados con la Salud

Décima Revisión

1/ S02,S12,S22,S32,S42,S52,S62,S72,S82,S92,T02,T08,T10,T12,T 142

Fuente: Sistema Automatizado de Egresos Hospitalarios en el país (SAEH) y Sistemas de información de los Institutos N acionales de Salud y del Hospital General de México 


\section{Principales causas de Mortalidad Hospitalaria, SSA. México, 2000}

Núm. de orden

Causa

Clave CIE-10

Egresos $\%$

Total

A00-Y98

$36531 \quad 100.0$

1

Ciertas afecciones originadas en el periodo perinatal

P00-P96 A33

7086

19.4

- Dificultad respiratoria del recién nacido y otros trastornos respiratorios

originados en el periodo perinatal

P22-P28

$100-151$ exc. 146

$2755 \quad 7.5$

2

126-145 $147-151$

$3145 \quad 8.6$

- Enfermedades de la circulación pulmonar y otras enfermedades del corazón

E10-E14

C00-C 97

Tumores malignos

- Leucemias

- Tumor maligno de la tráquea, de los bronquios y del pulmón

C91-C95

- Tumor maligno del cuello del útero

C 33 C 34

Enfermedades del hígado

$\mathrm{C} 53$

5

- Enfermedad alcohólica del hígado

$\mathrm{K} 70-\mathrm{K} 76$

$\mathrm{K} 70$

$160-169$

$1275 \quad 3.5$

Enfermedades cerebrovasculares

Q 00-Q 99

$2741 \quad 7.5$

$\begin{array}{r}6 \\ 7 \\ \hline\end{array}$

Malformaciones congénitas, deformidades y anomalías cromosómicas

Q 20-Q 28

$2588 \quad 7.1$

- Malformaciones congénitas del sistema circulatorio

V01-X 59 Y 40-Y 86

$\begin{array}{ll}476 & 1.3 \\ 209 & 0.6\end{array}$

\begin{tabular}{|c|c|c|c|c|}
\hline & - Malformaciones congénitas del sistema circulatorio & Q 20-Q 28 & 673 & 1.8 \\
\hline 8 & Accidentes & V01-X 59 Y 40-Y 86 & 1454 & 4.0 \\
\hline 9 & Influenza y N eumonía & J10-J18 & 1346 & 3.7 \\
\hline 10 & Insuficiencia renal & N 17-N 19 & 957 & 2.6 \\
\hline 11 & Septicemia & A40-A41 & 814 & 2.2 \\
\hline 12 & Enfermedades pulmonares obstructivas crónicas & J44 & 755 & 2.1 \\
\hline 13 & Enfermedad por virus de la inmunodeficiencia humana (SIDA) & B20-B24 & 713 & 2.0 \\
\hline 14 & Desnutrición y otras deficiencias nutricionales & E40-E64 & 441 & 1.2 \\
\hline 15 & Tuberculosis pulmonar & $1 /$ & 410 & 1.1 \\
\hline 16 & Síndrome de dependencia del alcohol & F102 & 342 & 0.9 \\
\hline 17 & Enfermedades infecciosas intestinales & A00-A09 & 324 & 0.9 \\
\hline 18 & Embarazo, parto y puerperio & $000-099$ A34 F53 M830 & 297 & 0.8 \\
\hline 19 & Pancreatitis aguda y otras enfermedades del páncreas & K85-K86 & 193 & 0.5 \\
\hline 20 & Agresiones (homicidio) & $X 85-Y 09$ & 154 & 0.4 \\
\hline
\end{tabular}

Síntomas, signos y hallazgos anormales clínicos y de laboratorio

no clasificados en otra parte

R00-R99

Las demás causas

Clave CIE-10: códigos de la Clasificación Estadística Internacional de Enfermedades y Problemas Relacionados con la Salud Décima Revisión

1/ A150-A153,A 157,A159,A160-A162,A167,A169

Fuente: Sistema Automatizado de Egresos Hospitalarios en el país (SAEH) y Sistemas de información de los Institutos N acionales de Salud y del Hospital General de México 


\section{Cuadro VIII \\ Morbilidad Hospitalaria SEgún CaUSA de egreso y grupos DE EDAD SEGÚn LISTA CONDENSADA SSA. MÉXICO, 2000}

\begin{tabular}{|c|c|c|c|c|c|c|c|c|}
\hline & & & & Grup & le edad & & & \\
\hline Causa & Total & $<1$ año & $1 \mathrm{a} 4$ & 5 a 14 & 15 a 44 & 45 a 64 & 65 y más & NE \\
\hline Total & 1469332 & 127416 & 61063 & 88995 & 950519 & 141444 & 99676 & 219 \\
\hline Enfermedades infecciosas intestinales & 25664 & 8702 & 6495 & 2352 & 4474 & 1997 & 1638 & 6 \\
\hline Tuberculosis & 3449 & 32 & 84 & 152 & 1699 & 931 & 550 & 1 \\
\hline Infección meningocócica & 15 & 3 & 1 & 2 & 7 & 0 & 2 & 0 \\
\hline Sífilis & 59 & 43 & 1 & 1 & 9 & 3 & 2 & 0 \\
\hline Infección gonocócica & 42 & 0 & 0 & 4 & 32 & 6 & 0 & 0 \\
\hline Fiebre del dengue & 172 & 3 & 19 & 70 & 70 & 8 & 2 & 0 \\
\hline Fiebre del dengue hemorrágico & 44 & 5 & 5 & 16 & 15 & 1 & 2 & 0 \\
\hline Paludismo & 107 & 4 & 5 & 21 & 63 & 13 & 1 & 0 \\
\hline Tumor maligno del estómago & 1103 & 1 & 0 & 0 & 210 & 510 & 382 & 0 \\
\hline Tumor maligno del colon & 566 & 0 & 0 & 5 & 155 & 229 & 177 & 0 \\
\hline Tumor de rectosigmoides, recto y ano & 465 & 0 & 3 & 3 & 175 & 156 & 128 & 0 \\
\hline Tumor de la tráquea, bronquios y pulmón & 1511 & 0 & 1 & 4 & 211 & 597 & 698 & 0 \\
\hline Tumor maligno de la mama & 3407 & 1 & 1 & 4 & 1501 & 1467 & 433 & 0 \\
\hline Tumor maligno del cuello del útero & 4594 & 1 & 3 & 4 & 1926 & 2020 & 640 & 0 \\
\hline Leucemias & 4483 & 57 & 795 & 1946 & 1261 & 280 & 144 & 0 \\
\hline Leiomioma uterino & 11525 & 0 & 0 & 6 & 7576 & 3816 & 127 & 0 \\
\hline Enfermedades de la glándula tiroides & 1617 & 56 & 21 & 33 & 851 & 507 & 149 & 0 \\
\hline Diabetes mellitus & 26017 & 7 & 52 & 423 & 5788 & 11897 & 7848 & 2 \\
\hline Desnutrición y otras deficiencias nutricionales & 2307 & 955 & 534 & 227 & 164 & 134 & 292 & 1 \\
\hline Trastornos mentales & 24521 & 30 & 103 & 418 & 18168 & 4876 & 924 & 2 \\
\hline Esclerosis múltiple & 147 & 0 & 0 & 13 & 108 & 24 & 2 & 0 \\
\hline Enfermedades del ojo y sus anexos & 11008 & 220 & 778 & 1241 & 2394 & 2590 & 3785 & 0 \\
\hline Enfermedades del oído y la apófisis mastoides & 2248 & 106 & 232 & 459 & 962 & 367 & 122 & 0 \\
\hline Enfermedades del sistema circulatorio & 41740 & 429 & 365 & 850 & 8564 & 13831 & 17695 & 6 \\
\hline Enfermedades reumáticas crónicas del corazón & 1087 & 3 & 2 & 21 & 381 & 501 & 179 & 0 \\
\hline Enfermedades hipertensivas & 5764 & 2 & 7 & 52 & 1240 & 2259 & 2204 & 0 \\
\hline Infarto agudo del miocardio & 2037 & 1 & 0 & 0 & 220 & 906 & 910 & 0 \\
\hline Enfermedades cerebrovasculares & 9095 & 108 & 45 & 119 & 1163 & 2631 & 5025 & 4 \\
\hline Várices de los miembros inferiores & 868 & 0 & 1 & 4 & 359 & 371 & 133 & 0 \\
\hline Infecciones respiratorias agudas & 16722 & 7895 & 5099 & 1776 & 956 & 413 & 581 & 2 \\
\hline N eumonía & 29522 & 12247 & 8076 & 1865 & 2130 & 1758 & 3443 & 3 \\
\hline Influenza & 36 & 6 & 4 & 7 & 9 & 3 & 7 & 0 \\
\hline Enfermedades crónicas de las amígdalas y adenoides & 4108 & 37 & 900 & 2500 & 625 & 42 & 4 & 0 \\
\hline Bronquitis crónica, enfisema y asma & 9439 & 550 & 2707 & 1919 & 1941 & 1182 & 1140 & 0 \\
\hline Enfermedades de los dientes y estructuras de sostén & 1255 & 9 & 320 & 278 & 505 & 99 & 44 & 0 \\
\hline Ulceras gástrica y duodenal & 1537 & 3 & 1 & 27 & 505 & 482 & 519 & 0 \\
\hline Enfermedades del apéndice & 26066 & 100 & 921 & 7784 & 14709 & 1891 & 652 & 9 \\
\hline Hernia de la cavidad abdominal & 28666 & 894 & 1821 & 2698 & 10120 & 8270 & 4863 & 0 \\
\hline Enfermedades del hígado & 10295 & 52 & 156 & 281 & 3711 & 4427 & 1663 & 5 \\
\hline Colelitiasis y colecistitis & 27606 & 0 & 1 & 145 & 17274 & 7075 & 3111 & 0 \\
\hline Enfermedades del sistema osteomuscular & & & & & & & & \\
\hline y tejido conjuntivo & 15514 & 159 & 793 & 2561 & 7080 & 3248 & 1673 & 0 \\
\hline Enfermedades del sistema urinario & 38228 & 461 & 946 & 3117 & 17754 & 10889 & 5057 & 4 \\
\hline
\end{tabular}

\section{(Continúa)}




\section{Cuadro VIII}

\section{(Continuación)}

\begin{tabular}{|c|c|c|c|c|c|c|c|c|}
\hline Hiperplasia de la próstata & 3658 & 0 & 0 & 0 & 59 & 1010 & 2589 & 0 \\
\hline Prolapso genital femenino & 5444 & 0 & 0 & 8 & 1279 & 2676 & 1481 & 0 \\
\hline Embarazo, parto y puerperio & 666475 & 0 & 0 & 5955 & 658839 & 1579 & 0 & 102 \\
\hline Aborto & 73958 & 0 & 0 & 683 & 72723 & 545 & 0 & 7 \\
\hline Parto único espontáneo & 348919 & 0 & 0 & 2812 & 345579 & 461 & 0 & 67 \\
\hline Las demás afecciones obstétricas directas & 243598 & 0 & 0 & 2460 & 240537 & 573 & 0 & 28 \\
\hline Ciertas afecciones originadas en el periodo perinatal & 67312 & 67302 & 6 & 1 & 2 & 0 & 1 & 0 \\
\hline Malformaciones congénitas & 23178 & 8080 & 5418 & 5596 & 3503 & 447 & 132 & 2 \\
\hline Síntomas, signos y hallazgos N CO P & 16642 & 1254 & 1876 & 2250 & 7346 & 2017 & 1862 & 37 \\
\hline Traumatismos y envenenamientos & 121402 & 2106 & 10625 & 25116 & 57224 & 15263 & 11056 & 12 \\
\hline Fracturas & 48130 & 398 & 2560 & 11951 & 19630 & 6781 & 6804 & 6 \\
\hline Luxaciones, esguinces y torceduras & 3706 & 30 & 120 & 477 & 2229 & 526 & 324 & 0 \\
\hline Traumatismos y heridas & 48767 & 884 & 3759 & 8696 & 26919 & 5805 & 2700 & 4 \\
\hline Q uemaduras y corrosiones & 5787 & 255 & 1876 & 1319 & 1751 & 399 & 187 & 0 \\
\hline \multicolumn{9}{|l|}{ Factores que influyen en la salud y contacto } \\
\hline con los servicios de salud & 29863 & 7038 & 632 & 1156 & 18604 & 1733 & 698 & 2 \\
\hline Atención para la anticoncepción & 12749 & 0 & 0 & 5 & 12583 & 156 & 3 & 2 \\
\hline Las demás causas & 159553 & 8568 & 11263 & 15701 & 69961 & 30680 & 23357 & 23 \\
\hline
\end{tabular}




\section{Morbilidad Hospitalaria por causa de egreso y sexo, según lista condensada SSA. México, 2000}

Causas

Total

Enfermedades infecciosas intestinales

Tuberculosis

Infección meningocócica

Sífilis

Infección gonocócica

Fiebre del dengue

Fiebre del dengue hemorrágico

Paludismo

Tumor maligno del estómago

Tumor maligno del colon

Tumor de rectosigmoides, recto y ano

Tumor de la tráquea, bronquios y pulmón

Tumor maligno de la mama

Tumor maligno del cuello del útero

Leucemias

Leiomioma uterino

Enfermedades de la glándula tiroides

Diabetes mellitus

Desnutrición y otras deficiencias nutricionales

Trastornos mentales

Esclerosis múltiple

Enfermedades del ojo y sus anexos

Enfermedades del oído y la apófisis mastoides

Enfermedades del sistema circulatorio

Enfermedades reumáticas crónicas del corazón

Enfermedades hipertensivas

Infarto agudo del miocardio

Enfermedades cerebrovasculares

Várices de los miembros inferiores

Infecciones respiratorias agudas

Neumonía

Influenza

Enfermedades crónicas de las amígdalas y adenoides

Bronquitis crónica, enfisema y asma

Enfermedades de los dientes y estructuras de sostén

Ulceras gástrica y duodenal

Enfermedades del apéndice

Hernia de la cavidad abdominal

Enfermedades del hígado

Colelitiasis y colecistitis

Enfermedades del sistema osteomuscular

y tejido conjuntivo

Enfermedades del sistema urinario

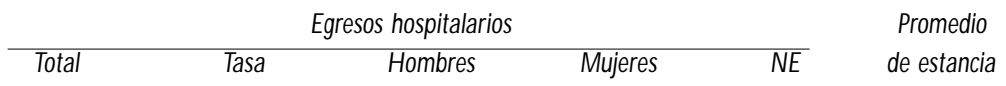

$1469332 \quad 3508.3$

395431

1073811

90

5.2

$25664 \quad 61$

3449

15

59

42

172

44

107

1103

566

465

1511

3407

4594

4483

11525

1617

26017

2307

24521

147

11008

2248

41740

1087

5764

2037

9095

868

16722

29522

36
4108

9439

1255

1537

26066

28666

10295

27606

61.3
8.2

13371

12293

1339

2110

0.0

0.1

0.1

0.4

0.1
0.3

2.6

0.3

2.6
1.4

1.4

1.4
1.1

1.1
3.6

3.6
8.1

8.1
11.0

10.7

27.5

3.9

62.1

5.5

58.5

0.4
26.3

26.3

5.4
99.7

99.7
2.6

2.6
13.8

13.8
4.9

21.7

2.1

39.9

70.5

0.1
9.8

9.8
22.5

22.5

3.0
3.7

3.7

62.2

62.2
68.4

24.6

65.9

15514

38228

37.0
91.3

7125
17355

7125
17355

\begin{tabular}{rr}
7125 & 8389 \\
17355 & 20871 \\
\hline
\end{tabular}

8389
20871

$\begin{array}{lll}8 & 0 & 9.7\end{array}$

$\begin{array}{rrr}8 & 0 & 13.5 \\ 28 & 0 & 9.6 \\ 33 & 0 & 2.8\end{array}$

$94 \quad 0 \quad 3.5$

$\begin{array}{lll}24 & 0 & 5.0 \\ 51 & 0 & 3.2\end{array}$

$\begin{array}{lll}499 & 0 & 7.8 \\ 292 & 0 & 8.9\end{array}$

292

238

238

547

547
4373
4594

4594

1941

11525

1368

15125

1137

8662

$\begin{array}{rrr}89 & 0 & 110.2\end{array}$

\begin{tabular}{rrr}
5932 & 0 & 10.9 \\
\hline & 0 & 1.8
\end{tabular}

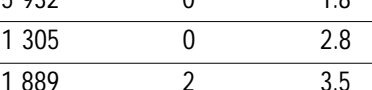

$\begin{array}{rrr}11889 & 2 & 3.5 \\ 729 & 0 & 13.2 \\ 3601 & 0 & 4.1\end{array}$

\section{(Continúa)}




\section{Cuadro IX}

\section{(Continuación)}

\begin{tabular}{|c|c|c|c|c|c|c|}
\hline Hiperplasia de la próstata & 3658 & 8.7 & 3658 & 0 & 0 & 5.2 \\
\hline Prolapso genital femenino & 5444 & 13.0 & 0 & 5444 & 0 & 3.5 \\
\hline Embarazo, parto y puerperio & 666475 & 1591.3 & 0 & 666475 & 0 & 1.6 \\
\hline Aborto & 73958 & 176.6 & 0 & 73958 & 0 & 1.5 \\
\hline Parto único espontáneo & 348919 & 833.1 & 0 & 348919 & 0 & 1.2 \\
\hline Las demás afecciones obstétricas directas & 243598 & 581.6 & 0 & 243598 & 0 & 2.3 \\
\hline Ciertas afecciones originadas en el periodo perinatal & 67312 & 160.7 & 37572 & 29701 & 39 & 6.7 \\
\hline Malformaciones congénitas & 23178 & 55.3 & 12976 & 10175 & 27 & 6.0 \\
\hline Síntomas, signos y hallazgos N C O P & 16642 & 39.7 & 7159 & 9483 & 0 & 3.5 \\
\hline Traumatismos y envenenamientos & 121402 & 289.9 & 81668 & 39730 & 4 & 4.8 \\
\hline Fracturas & 48130 & 114.9 & 32006 & 16123 & 1 & 5.3 \\
\hline Luxaciones, esguinces y torceduras & 3706 & 8.8 & 2479 & 1227 & 0 & 2.8 \\
\hline Traumatismos y heridas & 48767 & 116.4 & 36011 & 12754 & 2 & 4.3 \\
\hline Q uemaduras y corrosiones & 5787 & 13.8 & 3624 & 2163 & 0 & 9.4 \\
\hline \multicolumn{7}{|l|}{ Factores que influyen en la salud y contacto } \\
\hline con los servicios de salud & 29863 & 71.3 & 8285 & 21577 & 1 & 2.1 \\
\hline Atención para la anticoncepción & 12749 & 30.4 & 861 & 11888 & 0 & 1.1 \\
\hline Las demás causas & 159553 & 381.0 & 71640 & 87905 & 8 & 7.4 \\
\hline $\begin{array}{l}\text { Tasa por } 100000 \text { habitantes de población potencial } \\
\text { NE: no especificado } \\
\text { NCO P: no clasificado en otra parte }\end{array}$ & & & & & & \\
\hline
\end{tabular}




\section{Cuadro $X$ \\ Mortalidad Hospitalaria por causa de defunción y gRUpos de edad según lista condensada. Secretaría de Salud. México, 2000}

\begin{tabular}{|c|c|c|c|c|c|c|c|c|}
\hline \multirow[b]{2}{*}{ Causa } & \multirow[b]{2}{*}{ Total } & \multicolumn{6}{|c|}{ Grupos de edad } & \multirow[b]{2}{*}{$\mathrm{NE}$} \\
\hline & & $<1$ año & 1 a 4 & 5 a 14 & 15 a 44 & 45 a 64 & 65 y más & \\
\hline Total & 36531 & 9856 & 836 & 729 & 7048 & 7691 & 10350 & 21 \\
\hline Enfermedades infecciosas y parasitarias & 2690 & 385 & 148 & 73 & 1092 & 503 & 488 & 1 \\
\hline Enfermedades infecciosas intestinales & 324 & 168 & 53 & 8 & 29 & 25 & 41 & 0 \\
\hline Tuberculosis & 500 & 3 & 3 & 9 & 233 & 133 & 118 & 1 \\
\hline Tos ferina & 2 & 2 & 0 & 0 & 0 & 0 & 0 & 0 \\
\hline Otros tétanos & 27 & 0 & 0 & 1 & 8 & 9 & 9 & 0 \\
\hline Septicemia & 814 & 169 & 44 & 19 & 156 & 169 & 257 & 0 \\
\hline Paludismo & 1 & 0 & 0 & 0 & 0 & 1 & 0 & 0 \\
\hline Tumor maligno del estómago & 155 & 0 & 0 & 0 & 21 & 74 & 60 & 0 \\
\hline Tumor maligno del colon & 56 & 0 & 0 & 1 & 7 & 18 & 30 & 0 \\
\hline Tumor de rectosigmoides, recto y ano & 31 & 0 & 1 & 0 & 8 & 11 & 11 & 0 \\
\hline Tumor de la tráquea, bronquios y pulmón & 209 & 0 & 0 & 1 & 17 & 78 & 113 & 0 \\
\hline Tumor maligno de la mama & 166 & 0 & 0 & 0 & 52 & 83 & 31 & 0 \\
\hline Tumor maligno del cuello del útero & 171 & 0 & 0 & 0 & 54 & 83 & 34 & 0 \\
\hline Leucemias & 476 & 12 & 48 & 119 & 193 & 65 & 39 & 0 \\
\hline Anemias & 149 & 13 & 4 & 17 & 51 & 32 & 32 & 0 \\
\hline Diabetes mellitus & 2741 & 0 & 1 & 9 & 404 & 1117 & 1209 & 1 \\
\hline Desnutrición y otras deficiencias nutricionales & 441 & 100 & 44 & 21 & 49 & 40 & 187 & 0 \\
\hline Meningitis & 148 & 50 & 11 & 3 & 48 & 16 & 20 & 0 \\
\hline Enfermedades del sistema circulatorio & 5451 & 93 & 36 & 40 & 636 & 1513 & 3130 & 3 \\
\hline Fiebre reumática aguda & 4 & 0 & 0 & 0 & 4 & 0 & 0 & 0 \\
\hline Enfermedades reumáticas crónicas del corazón & 101 & 1 & 0 & 0 & 32 & 51 & 17 & 0 \\
\hline Enfermedades hipertensivas & 704 & 2 & 0 & 1 & 63 & 200 & 438 & 0 \\
\hline Infarto agudo del miocardio & 605 & 0 & 0 & 0 & 41 & 192 & 372 & 0 \\
\hline Enfermedades cerebrovasculares & 2006 & 24 & 8 & 14 & 242 & 584 & 1132 & 2 \\
\hline Aterosclerosis & 36 & 0 & 0 & 0 & 0 & 9 & 27 & 0 \\
\hline Infecciones respiratorias agudas & 120 & 21 & 6 & 0 & 31 & 14 & 48 & 0 \\
\hline N eumonía & 1344 & 233 & 88 & 39 & 227 & 202 & 552 & 3 \\
\hline Influenza & 2 & 0 & 0 & 1 & 0 & 1 & 0 & 0 \\
\hline Bronquitis crónica, enfisema y asma & 117 & 3 & 2 & 1 & 8 & 19 & 84 & 0 \\
\hline Ulceras gástrica y duodenal & 149 & 0 & 0 & 0 & 12 & 34 & 103 & 0 \\
\hline Enfermedades del apéndice & 54 & 1 & 2 & 5 & 4 & 12 & 30 & 0 \\
\hline Enfermedades del hígado & 2548 & 16 & 23 & 10 & 835 & 1200 & 460 & 4 \\
\hline Síndrome nefrítico, otras enfermedades glomerulares & 40 & 1 & 2 & 3 & 23 & 7 & 4 & 0 \\
\hline Hiperplasia de la próstata & 33 & 0 & 0 & 0 & 0 & 6 & 27 & 0 \\
\hline Embarazo, parto y puerperio & 299 & 0 & 0 & 3 & 293 & 3 & 0 & 0 \\
\hline Aborto & 14 & 0 & 0 & 0 & 14 & 0 & 0 & 0 \\
\hline Las demás afecciones obstétricas directas & 260 & 0 & 0 & 3 & 254 & 3 & 0 & 0 \\
\hline Ciertas afecciones originadas en el periodo perinatal & 7086 & 7085 & 0 & 0 & 0 & 0 & 0 & 1 \\
\hline Traumatismo del nacimiento & 43 & 43 & 0 & 0 & 0 & 0 & 0 & 0 \\
\hline Malformaciones congénitas & 1515 & 1225 & 130 & 52 & 85 & 15 & 7 & 1 \\
\hline Síntomas, signos y hallazgos N CO P & 547 & 111 & 17 & 19 & 141 & 126 & 131 & 2 \\
\hline Causas externas de morbilidad y mortalidad & 1868 & 39 & 60 & 81 & 876 & 424 & 386 & 2 \\
\hline Accidentes & 1454 & 33 & 50 & 70 & 623 & 336 & 342 & 0 \\
\hline Accidentes de tráfico de vehículos de motor & 337 & 4 & 5 & 18 & 204 & 58 & 48 & 0 \\
\hline Caídas & 318 & 10 & 13 & 12 & 89 & 86 & 108 & 0 \\
\hline Exposición al humo, fuego y llamas & 53 & 1 & 6 & 3 & 16 & 10 & 17 & 0 \\
\hline Envenenamiento accidental & 41 & 4 & 3 & 4 & 17 & 9 & 4 & 0 \\
\hline Lesiones autoinfligidas intencionalmente & 74 & 0 & 0 & 2 & 49 & 18 & 5 & 0 \\
\hline Agresiones (homicidio) & 154 & 2 & 5 & 1 & 97 & 35 & 14 & 0 \\
\hline Las demás & 7925 & 468 & 213 & 231 & 1881 & 1995 & 3134 & 3 \\
\hline
\end{tabular}




\section{Cuadro XI \\ Mortalidad Hospitalaria por causa de defunción y SEXo SEgún lista CONDENSAda. Secretaría de Salud. México, 2000}

\begin{tabular}{|c|c|c|c|c|c|}
\hline & & & es hospitala & & \\
\hline Causa & Total & Tasa & Hombres & Mujeres & $\mathrm{NE}$ \\
\hline Total & 36531 & 2486.2 & 20203 & 16317 & 11 \\
\hline Enfermedades infecciosas y parasitarias & 2690 & 183.1 & 1666 & 1024 & 0 \\
\hline Enfermedades infecciosas intestinales & 324 & 22.1 & 178 & 146 & 0 \\
\hline Tuberculosis & 500 & 34.0 & 340 & 160 & 0 \\
\hline Tos ferina & 2 & 0.1 & 1 & 1 & 0 \\
\hline O tros tétanos & 27 & 1.8 & 19 & 8 & 0 \\
\hline Septicemia & 814 & 55.4 & 396 & 418 & 0 \\
\hline Paludismo & 1 & 0.1 & 1 & 0 & 0 \\
\hline Tumor maligno del estómago & 155 & 10.5 & 76 & 79 & 0 \\
\hline Tumor maligno del colon & 56 & 3.8 & 29 & 27 & 0 \\
\hline Tumor de rectosigmoides, recto y ano & 31 & 2.1 & 19 & 12 & 0 \\
\hline Tumor de la tráquea, bronquios y pulmón & 209 & 14.2 & 140 & 69 & 0 \\
\hline Tumor maligno de la mama & 166 & 11.3 & 1 & 165 & 0 \\
\hline Tumor maligno del cuello del útero & 171 & 11.6 & 0 & 171 & 0 \\
\hline Leucemias & 476 & 32.4 & 232 & 244 & 0 \\
\hline Anemias & 149 & 10.1 & 71 & 78 & 0 \\
\hline Diabetes mellitus & 2741 & 186.5 & 1194 & 1547 & 0 \\
\hline Desnutrición y otras deficiencias nutricionales & 441 & 30.0 & 234 & 207 & 0 \\
\hline Meningitis & 148 & 10.1 & 85 & 63 & 0 \\
\hline Enfermedades del sistema circulatorio & 5451 & 371.0 & 2616 & 2835 & 0 \\
\hline Fiebre reumática aguda & 4 & 0.3 & 2 & 2 & 0 \\
\hline Enfermedades reumáticas crónicas del corazón & 101 & 6.9 & 28 & 73 & 0 \\
\hline Enfermedades hipertensivas & 704 & 47.9 & 300 & 404 & 0 \\
\hline Infarto agudo del miocardio & 605 & 41.2 & 334 & 271 & 0 \\
\hline Enfermedades cerebrovasculares & 2006 & 136.5 & 986 & 1020 & 0 \\
\hline Aterosclerosis & 36 & 2.5 & 19 & 17 & 0 \\
\hline Infecciones respiratorias agudas & 120 & 8.2 & 70 & 50 & 0 \\
\hline N eumonía & 1344 & 91.5 & 743 & 600 & 1 \\
\hline Influenza & 2 & 0.1 & 1 & 1 & 0 \\
\hline Bronquitis crónica, enfisema y asma & 117 & 8.0 & 62 & 55 & 0 \\
\hline Ulceras gástrica y duodenal & 149 & 10.1 & 90 & 59 & 0 \\
\hline Enfermedades del apéndice & 54 & 3.7 & 30 & 24 & 0 \\
\hline Enfermedades del hígado & 2548 & 173.4 & 1874 & 674 & 0 \\
\hline Síndrome nefrítico, otras enfermedades glomerulares & 40 & 2.7 & 26 & 14 & 0 \\
\hline Hiperplasia de la próstata & 33 & 2.2 & 33 & 0 & 0 \\
\hline Embarazo, parto y puerperio & 299 & 20.3 & 0 & 299 & 0 \\
\hline Aborto & 14 & 1.0 & 0 & 14 & 0 \\
\hline Las demás afecciones obstétricas directas & 260 & 17.7 & 0 & 260 & 0 \\
\hline Ciertas afecciones originadas en el periodo perinatal & 7086 & 482.3 & 4111 & 2974 & 1 \\
\hline Traumatismo del nacimiento & 43 & 2.9 & 23 & 20 & 0 \\
\hline Malformaciones congénitas & 1515 & 103.1 & 797 & 710 & 8 \\
\hline Síntomas, signos y hallazgos N CO P & 547 & 37.2 & 321 & 226 & 0 \\
\hline Causas externas de morbilidad y mortalidad & 1868 & 127.1 & 1431 & 436 & 1 \\
\hline Accidentes & 1454 & 99.0 & 1103 & 350 & 1 \\
\hline Accidentes de tráfico de vehículos de motor & 337 & 22.9 & 263 & 74 & 0 \\
\hline Caídas & 318 & 21.6 & 232 & 86 & 0 \\
\hline Exposición al humo, fuego y llamas & 53 & 3.6 & 33 & 20 & 0 \\
\hline Envenenamiento accidental & 41 & 2.8 & 27 & 14 & 0 \\
\hline Lesiones auto infligidas intencionalmente & 74 & 5.0 & 53 & 21 & 0 \\
\hline Agresiones (homicidio) & 154 & 10.5 & 126 & 28 & 0 \\
\hline Las demás & 7925 & 539.4 & 4251 & 3674 & 0 \\
\hline $\begin{array}{l}\text { Tasa por } 100000 \text { egresos hospitalarios } \\
\text { N E: no especificado } \\
\text { N CO P: no clasificado en otra parte }\end{array}$ & & & & & \\
\hline
\end{tabular}




\section{Cuadro XII \\ Total de INTERVENCIONES QUiRÚRgicas Por CAPítulo, SEgúN SEXo, eN hospitales de La SSA. México, 2000}

Clave CIE-9MC Intervenciones quirúrgicas

\begin{tabular}{|c|c|c|c|c|c|c|c|c|c|}
\hline \multicolumn{3}{|c|}{ Total } & \multicolumn{3}{|c|}{ Hombres } & \multicolumn{3}{|c|}{ M ujeres } & NE \\
\hline Núm. & $\%$ & $\%$ & Núm. & $\%$ & $\%$ & Núm. & $\%$ & $\%$ & Núm. \\
\hline 724046 & 100.0 & 100.0 & 127791 & 17.6 & 100.0 & 596250 & 82.3 & 100.0 & 5 \\
\hline
\end{tabular}

\begin{tabular}{|c|c|c|c|c|c|c|c|c|c|c|c|}
\hline $01-86$ & Total & 724046 & 100.0 & 100.0 & 127791 & 17.6 & 100.0 & 596250 & 82.3 & 100.0 & 5 \\
\hline $01-05$ & Del sistema nervioso & 6420 & 100.0 & 0.9 & 3786 & 59.0 & 3.0 & 2634 & 41.0 & 0.4 & 0 \\
\hline $06-07$ & Del sistema endócrino & 1088 & 100.0 & 0.2 & 302 & 27.8 & 0.2 & 786 & 72.2 & 0.1 & 0 \\
\hline 08-16 & De los ojos & 11186 & 100.0 & 1.5 & 5356 & 47.9 & 4.2 & 5830 & 52.1 & 1.0 & 0 \\
\hline $18-20$ & Del oído & 1666 & 100.0 & 0.2 & 805 & 48.3 & 0.6 & 861 & 51.7 & 0.1 & 0 \\
\hline $21-29$ & De la nariz, de la boca y de la faringe & 13537 & 100.0 & 1.9 & 7311 & 54.0 & 5.7 & 6226 & 46.0 & 1.0 & 0 \\
\hline $30-34$ & Del aparato respiratorio & 3983 & 100.0 & 0.6 & 2857 & 71.7 & 2.2 & 1126 & 28.3 & 0.2 & 0 \\
\hline $35-39$ & Del aparato cardiovascular & 4802 & 100.0 & 0.7 & 2220 & 46.2 & 1.7 & 2581 & 53.7 & 0.4 & 1 \\
\hline $40-41$ & Del sistema hemático y linfático & 713 & 100.0 & 0.1 & 403 & 56.5 & 0.3 & 310 & 43.5 & 0.1 & 0 \\
\hline $42-54$ & Del aparato digestivo & 99986 & 100.0 & 13.8 & 42852 & 42.9 & 33.5 & 57132 & 57.1 & 9.6 & 2 \\
\hline $55-59$ & Del aparato urinario & 6169 & 100.0 & 0.9 & 3182 & 51.6 & 2.5 & 2987 & 48.4 & 0.5 & 0 \\
\hline $60-64$ & De los órganos genitales masculinos & 10838 & 100.0 & 1.5 & 10838 & 100.0 & 8.5 & 0 & 0.0 & 0.0 & 0 \\
\hline $65-71$ & De los órganos genitales femeninos & 200877 & 100.0 & 27.7 & 0 & 0.0 & 0.0 & 200877 & 100.0 & 33.7 & 0 \\
\hline $72-75$ & Procedimientos obstétricos & 280306 & 100.0 & 38.7 & 0 & 0.0 & 0.0 & 280306 & 100.0 & 47.0 & 0 \\
\hline $76-84$ & Del aparato musculoesquelético & 56303 & 100.0 & 7.8 & 35470 & 63.0 & 27.8 & 20833 & 37.0 & 3.5 & 0 \\
\hline $85-86$ & Del aparato tegumentario & 26172 & 100.0 & 3.6 & 12409 & 47.4 & 9.7 & 13761 & 52.6 & 2.3 & 2 \\
\hline
\end{tabular}

Clave CIE-9MC: corresponde a los códigos de la Clasificación de Procedimientos (Volumen 3) de la Modificación Clínica de la C IE-9

Se excluyen códigos de procedimientos considerados como diagnósticos y terapéuticos no quirúrgicos

NE: no especificado

Sólo comprende a los hospitales que utilizan el Sistema Automatizado de Egresos Hospitalarios (SAEH)

$\mathrm{N}$ o incluye a los Institutos N acionales de Salud, el Hospital General de México ni hospitales de la SSA del DDF 


\section{Total de intervenciones Quirúrgicas por CApítulo y GRUPos de edAd, SSA. México, 2000}

\begin{tabular}{|c|c|c|c|c|c|c|c|c|c|}
\hline Clave CIE-9MC & Intervenciones quirúrgicas & Total & $<1$ & $1-4$ & $5-14$ & $15-44$ & $45-64$ & $65 y+$ & Noe. \\
\hline \multicolumn{10}{|l|}{ Número } \\
\hline $01-86$ & Total & 724046 & 7654 & 14615 & 42417 & 573275 & 54916 & 31101 & 68 \\
\hline $01-05$ & Del sistema nervioso & 6420 & 1107 & 457 & 824 & 2440 & 979 & 613 & 0 \\
\hline $06-07$ & Del sistema endócrino & 1088 & 4 & 50 & 117 & 592 & 257 & 68 & 0 \\
\hline $08-16$ & De los ojos & 11186 & 130 & 600 & 1205 & 2566 & 2613 & 4072 & 0 \\
\hline $18-20$ & Del oído & 1666 & 36 & 101 & 627 & 728 & 131 & 43 & 0 \\
\hline $21-29$ & De la nariz, de la boca y de la faringe & 13537 & 809 & 2419 & 4201 & 5209 & 649 & 250 & 0 \\
\hline $30-34$ & Del aparato respiratorio & 3983 & 258 & 219 & 248 & 1997 & 758 & 503 & 0 \\
\hline $35-39$ & Del aparato cardiovascular & 4802 & 876 & 288 & 388 & 1630 & 1051 & 569 & 0 \\
\hline $40-41$ & Del sistema hemático y linfático & 713 & 24 & 51 & 117 & 365 & 106 & 50 & 0 \\
\hline $42-54$ & Del aparato digestivo & 99986 & 3011 & 3417 & 11185 & 52679 & 19606 & 10073 & 15 \\
\hline $55-59$ & Del aparato urinario & 6169 & 104 & 270 & 504 & 2545 & 1595 & 1151 & 0 \\
\hline $60-64$ & De los órganos genitales masculinos & 10838 & 288 & 1918 & 2672 & 2617 & 1265 & 2078 & 0 \\
\hline $65-71$ & De los órganos genitales femeninos & 200877 & 6 & 4 & 972 & 185723 & 11860 & 2291 & 21 \\
\hline $72-75$ & Procedimientos obstétricos & 280306 & 0 & 0 & 2818 & 277068 & 391 & 0 & 29 \\
\hline $76-84$ & Del aparato musculoesquelético & 56303 & 653 & 3321 & 12999 & 22659 & 9177 & 7492 & 2 \\
\hline $85-86$ & Del aparato tegumentario & 26172 & 348 & 1500 & 3540 & 14457 & 4478 & 1848 & 1 \\
\hline \multicolumn{10}{|c|}{ Distribución porcentual } \\
\hline $01-86$ & Total & 100.0 & 1.1 & 2.0 & 5.9 & 79.2 & 7.6 & 4.3 & 0.0 \\
\hline $01-05$ & Del sistema nervioso & 100.0 & 17.2 & 7.1 & 12.8 & 38.0 & 15.2 & 9.5 & 0.0 \\
\hline $06-07$ & Del sistema endócrino & 100.0 & 0.4 & 4.6 & 10.8 & 54.4 & 23.6 & 6.3 & 0.0 \\
\hline 08-16 & De los ojos & 100.0 & 1.2 & 5.4 & 10.8 & 22.9 & 23.4 & 36.4 & 0.0 \\
\hline $18-20$ & Del oído & 100.0 & 2.2 & 6.1 & 37.6 & 43.7 & 7.9 & 2.6 & 0.0 \\
\hline $21-29$ & De la nariz, de la boca y de la faringe & 100.0 & 6.0 & 17.9 & 31.0 & 38.5 & 4.8 & 1.8 & 0.0 \\
\hline $30-34$ & Del aparato respiratorio & 100.0 & 6.5 & 5.5 & 6.2 & 50.1 & 19.0 & 12.6 & 0.0 \\
\hline $35-39$ & Del aparato cardiovascular & 100.0 & 18.2 & 6.0 & 8.1 & 33.9 & 21.9 & 11.8 & 0.0 \\
\hline $40-41$ & Del sistema hemático y linfático & 100.0 & 3.4 & 7.2 & 16.4 & 51.2 & 14.9 & 7.0 & 0.0 \\
\hline $42-54$ & Del aparato digestivo & 100.0 & 3.0 & 3.4 & 11.2 & 52.7 & 19.6 & 10.1 & 0.0 \\
\hline $55-59$ & Del aparato urinario & 100.0 & 1.7 & 4.4 & 8.2 & 41.3 & 25.9 & 18.7 & 0.0 \\
\hline $60-64$ & De los órganos genitales masculinos & 100.0 & 2.7 & 17.7 & 24.7 & 24.1 & 11.7 & 19.2 & 0.0 \\
\hline $65-71$ & De los órganos genitales femeninos & 100.0 & 0.0 & 0.0 & 0.5 & 92.5 & 5.9 & 1.1 & 0.0 \\
\hline $72-75$ & Procedimientos obstétricos & 100.0 & 0.0 & 0.0 & 1.0 & 98.8 & 0.1 & 0.0 & 0.0 \\
\hline $76-84$ & Del aparato musculoesquelético & 100.0 & 1.2 & 5.9 & 23.1 & 40.2 & 16.3 & 13.3 & 0.0 \\
\hline $85-86$ & Del aparato tegumentario & 100.0 & 1.3 & 5.7 & 13.5 & 55.2 & 17.1 & 7.1 & 0.0 \\
\hline
\end{tabular}

Clave CIE-9MC: corresponde a los códigos de la Clasificación de Procedimientos (Volumen 3) de la Modificación Clínica de la CIE-9

Se excluyen códigos de procedimientos considerados como diagnósticos y terapéuticos no quirúrgicos

Sólo comprende a los hospitales que utilizan el SistemaAutomatizado de Egresos Hospitalarios (SAEH)

No incluye a los Institutos N acionales de Salud, el Hospital General de México ni hospitales de la SSA del DDF

Comprende la primera, segunda y tercera cirugías 


\section{Cuadro XIV \\ Principales intervenciones Quirúrgicas, SSA. México, 2000}

\begin{tabular}{|c|c|c|c|c|}
\hline \multirow[t]{2}{*}{ Orden } & Procedimiento & Clave Vol. 3 de la CIE-9MC & Núm. & $\%$ \\
\hline & Total & & 724046 & 100.0 \\
\hline 1 & Cesárea & $740-742 \quad 744-749$ & 145679 & 20.1 \\
\hline 2 & Episiotomía & $736 X$ & 103656 & 14.3 \\
\hline 3 & Destrucción u oclusión bilateral de las trompas de Falopio & 662663665 & 82839 & 11.4 \\
\hline 4 & Dilatación y legrado uterino & 690 & 60202 & 8.3 \\
\hline 5 & Reducción de fractura & $767790-795$ & 28605 & 4.0 \\
\hline 6 & Hernioplastía de la cavidad abdominal & $530-539$ & 24474 & 3.4 \\
\hline 7 & A pendicectomía & 470471 & 23586 & 3.3 \\
\hline 8 & Colecistectomía & 512 & 19527 & 2.7 \\
\hline 9 & Exploración manual de la cavidad uterina después del parto & $757 X$ & 16779 & 2.3 \\
\hline 10 & Histerectomía & $683-687689$ & 15571 & 2.2 \\
\hline 11 & Laparotomía & 541 & 14133 & 2.0 \\
\hline 12 & Reparación de la vulva o el perineo & 717 & 10209 & 1.4 \\
\hline 13 & Extirpación o destrucción, de lesión o tejido de piel y tejido subcutáneo & 862 & 6727 & 0.9 \\
\hline 14 & Extracción de cristalino y/o inserción de prótesis (catarata) & 131-139 & 5785 & 0.8 \\
\hline 15 & Amputaciones y desarticulaciones & $8408418491-8493$ & 4807 & 0.7 \\
\hline 16 & Injerto y reparación de piel y tejido subcutáneo & $866-868$ & 4119 & 0.6 \\
\hline 17 & Fórceps durante el parto & $720-72472517253726$ & 3661 & 0.5 \\
\hline 18 & Sutura de piel y tejido subcutáneo & 865 & 3627 & 0.5 \\
\hline 19 & Amigdalectomía y adenoidectomía & $282-286$ & 3582 & 0.5 \\
\hline 20 & Circuncisión & $640 \mathrm{X}$ & 3080 & 0.4 \\
\hline & Los demás procedimientos & & 143398 & 19.8 \\
\hline
\end{tabular}

Clave CIE-9MC: corresponde a los códigos de la Clasificación de Procedimientos (Volumen 3) de la Modificación Clínica de la CIE-9

Se excluyen códigos de procedimientos considerados como diagnósticos y terapéuticos no quirúrgicos

Fuente: Subsistema Automatizado de Egresos Hospitalarios (SAEH)

Sólo comprende a los hospitales que utilizan el Sistema Automatizado de Egresos Hospitalarios (SAEH)

No incluye Institutos N acionales de Salud, Hospital General de México ni hospitales de la SSA del DDF 